Ann. Biol. anim. Bioch. Biophys., 1972, 12 (4), 6r7-63o.

\title{
INFLUENCE DE L'ACIDE CAPRYLIQUE SUR LE MÉTABOLISME DES AUTRES AGIDES GRAS CHEZ LE RAT EN CROISSANCE
}

\author{
B. AUROUSSEAU et L. DE GROOT \\ avec la collaboration technique de Françoise Duborsset et de J. BEJor \\ Laboratoire d'Études des Métabolismes, \\ Centre de Recherches de Clermont-Ferrand, I. N. R. A., \\ Theix 63110 Beaumont
}

\section{RÉSUMÉ}

L'influence de l'acide caprylique sur le métabolisme des acides gras a été étudiée chez le Rat en croissance, en complément d'une étude de l'utilisation énergétique de cet acide réalisée au moyen de la méthode d'abattage et d'analyse des carcasses (AURousSEAU, 1972). L'acide caprylique était incorporé aux régimes par substitution à des quantités isoénergétiques de l'amidon d'un régime témoin comportant par ailleurs ro p. Ioo d'énergie sous forme d'acides gras longs (insaturés dans l'exp. I, en mélange dans l'exp. II). La première expérience a permis de tester l'influence de l'acide caprylique à deux taux différents $(25,8$ et $28,2 \mathrm{p}$. Ioo de l'énergie métabolisable du régime) et pour 3 niveaux d'alimentation différents ("élevé ", " normal " ou " restreint "). La seconde expérience a permis de comparer l'influence de l'acide caprylique à celles des acides laurique et myristique et d'en étudier les interactions.

L'ingestion d'acide caprylique, laurique ou myristique, conformément à des résultats antérieurs, est suivie d'un enrichissement des lipides corporels en acides gras à chaîne moyenne et d'un accroissement relatif des activités de synthèse ou d'élongation d'acides gras par rapport aux activités de désaturation. De plus, l'acide caprylique exerce une action d' "épargne " sur les acides gras à chaîne plus longue et favorise en particulier le dépôt des acides gras à I2, 14 ou I6 atomes de carbone.

L'influence de l'acide caprylique peut être contrebalancée en totalité par des acides gras longs apportés par le régime (exp. II).

Ces résultats sont discutés en fonction des stades intermédiaires du métabolisme et de l'âge physiologique des animaux expérimentaux. Dans ces conditions, il apparaît comme vraisemblable que l'incorporation aux aliments d'allaitement d'acides gras à chaîne moyenne puisse favoriser l'amélioration de la qualité des carcasses des jeunes animaux domestiques.

\section{INTRODUCTION}

Dans le cadre des études poursuivies au laboratoire sur la nutrition énergétique du jeune monogastrique en croissance intensive, la connaissance de l'utilisation métabolique des nutriments, et en particulier des acides gras de longueur et de nature 
de chaîne đifférentes, peut contribuer à l'interprétation et à la compréhension de l'effet global qu'ils exercent sur l'utilisation de l'énergie des régimes ainsi qu'à la maîtrise de la qualité des carcasses et des dépôts adipenx des animaux domestiques.

Ainsi, chez le Rat, 1'acide caprylique est rapidement métabolisé en éléments à deux carbones (BRADy et GuRIN, I950) et oxydé en CO (METAIs et BACr, I967). Le catabolisme oxydatif des acides laurique et myristique est également très rapide (KRISCHNER et HARRIS, I96I ; MÉTAIS et al., I967). En addition à cet effet, les acides gras à chaîne moyenne semblent accroître chez le Rat la teneur des dépôts adipeux corporels en acides gras saturés (Bol,INGER et REISER, I865; KAUNITZ et JoHNSON, I968). Or l'accroissement de la teneur en acides gras saturés des dépôts adipeux sous-cutanés s'accompagne, en particulier chez l'Agneau, d'une baisse de leur teneur en eau et d'une amélioration de leur qualité de présentation et de fermeté (AURoussEAu et al., I972).

Au cours d'études sur rats, nous avons d'abord pu mettre en évidence que l'ingestion d'acide caprylique à égalité d'ingestion d'énergie métabolisable (EM) induisait une réduction de la quantité totale de lipides fixés et un accroissement de la teneur des lipides corporels totaux en acides gras à chaîne moyenne et en acides gras saturés (de Groo's et al., I970).

Dans une seconde phase, la réalisation de bilans précis d'acides gras nous a permis d'observer des phénomènes sensiblement différents au cours d'une expérience où les apports alimentaires d'acide oléique (Io p. Ioo de EM) ont masqué l'influence de l'acide caprylique (25 p. Ioo de l'EM du régime). Dans ces conditions, l'apport d'acide caprylique a entraîné une réduction de I4 p. Ioo de la quantité d'acides gras totaux déposés en 30 jours et cette réduction a affecté diversement les quantités d'acides gras individuels déposés : diminution de I9 p. Ioo dans le cas des acides gras saturés et de ro p. Ioo dans les cas de l'acide oléique (Aurousseau et al., r970). Au cours de cette même expérience un apport inférieur d'acide caprylique ( 5 p. Ioo de EM du régime) a exercé sur le métabolisme lipidique une influence comparable à celle observée au cours de nos premiers essais : dans ces conditions, la quantité d'acides gras totaux déposés en 30 jours a été réduite de $2 \mathrm{p}$. Ioo, la quantité d'acides gras longs saturés déposés pendant le même temps accrue de $6 \mathrm{p}$. Ioo et la quantité correspondante d'acide oléique réduite de $4 \mathrm{p}$. Ioo. Des mesures complémentaires de catabolisme oxydatif nous ont permis de mettre en évidence une exagération du cycle lipolyselipogenèse : pour le régime témoin " glucides " et le régime comportant $\mathrm{I}_{5} \mathrm{p}$. Ioo de l'EM sous forme d'acide caprylique, la couverture des besoins en énergie thermique par le catabolisme direct des nutriments est respectivement de 90 et 80 p. Ioo, et après 30 jours d'essais, on ne retrouve dans les dépôts des animaux ayant reçu ces régimes que 78 et $69 \mathrm{p}$. Ioo de l'acide oléîque absorbé, alors que I7 p. Ioo seulement sont directement catabolisés (AUROUSSEAU et al., I970).

En complément de ces premiers travaux, nous avons entrepris l'étude del'influence du niveau d'ingestion et de la teneur en azote des régimes sur l'utilisation de l'énergie des acides gras à chaîne moyenne (Aurousseau, 1972), et sur le métabolisme lipidique du rat en croissance .Cette étude a été réalisée au cours de deux expériences successives (exp. I et exp. II). 


\section{MATÉRIEL, ET MÉTHODES}

Des rats mâles de souche Wistar, sevrés au poids moyen de $45 \mathrm{~g}$ (2 I jours d'âge) proviennent du Centre de Sélection et d'Élevage d'animaux de laboratoire du C. N. R. S. Ils reçoivent pendant 4 jours un aliment standard du commerce avant d'être répartis en hots homologues de I 5 animaux, sur la base du poids et du gain de poids observé au cours de cette période pré-expérimentale, ils sont mis en expérience à un âge moyen de 30 jours ( $70 \mathrm{~g}$ de poids vif).

En fin d'expérience, on conserve dans chaque lot 9 à I 5 animaux dont les ingestions d'énergie métabolisables sont voisines $( \pm 5 \mathrm{p}$. I00)

L'expérience I a utilisé, sur une période de 25 jours, trois régimes à forte teneur en azote $(6,2 \mathrm{mg}$ azote digestible/kcal EM), deux régimes comportant de l'acide caprylique (tricapryline) aux niveaux de $30 \mathrm{p}$. Ioo de EM du régime (régime "Tri $\mathrm{C}_{8} 30 \mathrm{p}$. Ioo ", lots $\mathrm{A}_{1}$ et $\mathrm{A}_{2}$ ) et de $25 \mathrm{p}$. Ioo de EM du régime (régime "Tri $\mathrm{C}_{8} 25 \mathrm{p}$. Ioo ", lots $\mathrm{B}_{1}, \mathrm{~B}_{2}$ et $\mathrm{B}_{3}$ ) et un régime témoin ( glucides \#, lots $\mathrm{C}_{1}, \mathrm{C}_{2}$ et $\mathrm{C}_{3}$ ). Ces régimes ont été offerts à différents niveaux d'ingestion : 3 lots d'animaux présentant spontanément un haut niveau d'ingestion (lots $A_{1}, B_{1}$, et $C_{1}$ ), 3 lots d'animaux présentant des niveaux d'ingestion normaux" (lots $A_{2}, B_{2}$ et $C_{2}$ ) et deux lots soumis à des restrictions alimentaires de Io p. Ioo $\left(\operatorname{lot} \mathbf{B}_{3}\right.$ et $\left.C_{3}\right)$.

L'expérience II a utilisé sur une période de $2 \mathrm{I}$ jours, 4 régimes à teneur moyenne en azote $\left(5,4 \mathrm{mg}\right.$ azote digestible/kcal EM.) : un régime témoin " glucides " (lot $\mathrm{G}$ ), un régime "Tri $\mathrm{C}_{\mathrm{B}}$ I5 p. Ioo" (lot F) comportant de l'acide caprylique (tricapryline) au niveau de I 5 p. roo de EM, un régime "Coprah $\mathrm{I}_{5} \mathrm{p}$. IOo "(lot $\mathrm{E}$ ), dans lequel un mélange d'acides gras à chaîne moyenne (I I p. roo de $\mathrm{C}_{8}, 8$ p. Ioo de $\mathrm{C}_{10}, 5^{8} \mathrm{p}$. Ioo de $\mathrm{C}_{12}$ et $22 \mathrm{p}$. Ioo de $\mathrm{C}_{14}$ ) étaient apportés au niveau de E. M. par de l'huile de Coprah, et, enfin, un régime "Coprah Tri $\mathrm{C}_{8} 20 \mathrm{p}$. 100 " (lot D) comportant I 5 p. Ioo de EM sous forme d'acide caprylique et I 5 P. Ioo de EM sous forme des acides gras à chaîne moyenne de l'huile de Coprah.

Les régimes de l'expérience I comportaient de l'huile d'arachide (de façon à apporter ro p. roo de l'EM sous forme d'acides gras). Dans la crainte que l'acide linoléique, fortement catabolisé, ne vienne interférer avec l'utilisation métabolique de l'acide caprylique nous avons utilisé dans l'expérience II de l'huile de palme dont nous avons réduit l'apport pour tenir compte des acides gras longs de l'huile de Coprah des régimes ingérés par les lots D et $\mathrm{E}$. Le détail des régimes ainsi que le détail du mode opératoire utilisé pour le broyage des animaux sont donnés dans une communication précédente (AURousseau, 1972).

En raison du rôle important que joue le foie dans le métabolisme de l'acide caprylique (BezARD et al., I966, Clement et al., I969), ces deux expériences ont été complétées par l'analyse du contenu en lipides et en acides gras de foies prélevés au cours d'une expérience précédente (AURoussEAU et al, I970).

Pour apprécier le métabolisme lipidique des animaux expérimentaux, nous avons procédé à des bilans d'acides gras : les lipides des aliments, des fèces, des carcasses et des foies ont été extraits après hydrolyse acide, selon la méthode employée par Toullec et al., (1968). Les acides gras ont été pesés après purification des extraits lipidiques par saponification. Leur composition centésimale a été déterminée par chromatographie en phase gazeuse de leurs esters méthyliques. La colonne utilisée avait une longueur de $2 \mathrm{~m}$ et un diamètre de $4 \mathrm{~mm}$ et était garnie d'un support (Anakrom $\mathrm{A}, 80 \mathrm{mesh}$ ) imprégné de I $8 \mathrm{p}$. Ioo de succinate de diéthylène glycol (DEGS). La température de la colonne était de $\mathrm{I} 60^{\circ} \mathrm{C}$, la mesure de la surface des pics était réalisée par un intégrateur Disc.

Au cours de l'expérience I, une série d'incidents techniques a empêché d'obtenir une bonne précision sur le bilan d'acides gras entaché d'une incertitude consécutive à un séchage défectueux des échantillons, ceci s'est traduit par des erreurs atteignant + 1o p. Ioo. La composition centésimale en acides gras individuels, par contre, a été déterminée avec une meilleure précision : les résultats obtenus sont la moyenne de quatre chromatographies de 2 échantillons différents et la reproductibilité est de 0,6 point.

Au cours de la seconde expérience, le bilan d'acides gras a été déterminé dans de bonnes conditions $( \pm 0,4$ p. Ioo) de même que la composition centésimale en acides gras (reproductibilité $\pm 0,6$ point).

\section{RÉSULTATS}

Au cours de l'expérience $I$, les régimes comportant de l'acide caprylique ont été moins bien acceptés que les régimes témoins. De ce fait les consommations des lots $A_{1}$ et $B_{1}$ (niveau d'ingestion élevé) ont été du même ordre de grandeur que celles du lot 
$C_{2}$ (niveau d'ingestion normal). De même, les consommations du lot $B_{2}$ (niveau d'ingestion normal) ont été du même ordre de grandeur que celles du lot $\mathrm{C}_{3}$ (niveau d'ingestion " restreint "), reproduisant ainsi les conditions dans lesquelles nous comparons habituellement 1'utilisation des régimes expérimentaux à celle des régimes témoins.

Au cours de cette expérience, l'ingestion d'acide caprylique a eu sur la quantité d'acides gras fixés un effet variable et comparable à celui sur la quantité d'énergie fixée (AURousseau, I972). Ainsi, pour des quantités de EM ingérée sensiblement égales (tabl. I) la fixation de lipides ou d'acides gras a été accrue de II à I4 p. Ioo

\section{TABLEAU I}

Bilans d'énergie et d'acides gras observés au cours de l'expérience I

\begin{tabular}{|c|c|c|c|c|c|c|}
\hline $\begin{array}{c}\text { Niveau relatif } \\
\text { d'ingestion } \\
(\%)\end{array}$ & $\begin{array}{c}\text { Lots } \\
\text { expérim. }\end{array}$ & Régimes & $\begin{array}{c}\text { E fixée } \\
\text { (kcal) }\end{array}$ & $\mid \begin{array}{c}\text { E fixée } \\
\text { sous forme de } \\
\left.\text { protéines ( }{ }^{1}\right) \\
\text { (kcal) }\end{array}$ & $\begin{array}{c}E \text { fixée } \\
\text { sous forme de } \\
\text { lipides }\left(^{1}\right) \\
\text { (kcal) }\end{array}$ & $\begin{array}{l}\text { E fixée } \\
\text { sous forme } \\
\text { d'AG }\left(^{2}\right) \\
\text { (kcal) }\end{array}$ \\
\hline \multirow[t]{2}{*}{90} & $A_{2}$ & $" \operatorname{Tri} \mathrm{C}_{8} 30 \% "$ & 292 & 148 & 144 & 127 \\
\hline & $\mathbf{B}_{3}$ & $" \operatorname{Tri} \mathrm{C}_{8} 25 \% "$ & 297 & 144 & 153 & 132 \\
\hline \multirow{2}{*}{100} & $\mathrm{~B}_{2}$ & $" \operatorname{Tri} \mathrm{C}_{8} 25 \% "$ & 332 & 168 & 164 & 140 \\
\hline & $\mathrm{C}_{3}$ & "GIucides" & 352 & 173 & 179 & 150 \\
\hline \multirow{3}{*}{107} & $A_{1}$ & $" \operatorname{Tri} \mathrm{C}_{8} 30 \% "$ & 372 & 173 & 199 & 173 \\
\hline & $\mathrm{B}_{1}$ & $" \operatorname{Tri} \mathrm{C}_{8} 25 \% "$ & 383 & 175 & 208 & 179 \\
\hline & $\mathrm{C}_{2}$ & "Glucides " & 372 & 182 & 190 & 158 \\
\hline 117 & $C_{1}$ & "Glucides" & 452 & 193 & 269 & 221 \\
\hline
\end{tabular}

(1) L'énergie fixée sous forme de protéines est obtenue par calcul à partir du bilan d'azote. Par différence avec le bilan énergétique on en déduit l'énergie fixée sous forme de lipides.

$\left({ }^{2}\right)$ La quantité d'acides gras fixés a été déterminée par extraction et l'énergie correspondante calculée en adoptant une valeur de $9,5 \mathrm{kcal} / \mathrm{g}$ d'acides gras.

environ, respectivement chez les animaux des lots $\mathrm{A}_{1}$ (régime " $\operatorname{Tri} \mathrm{C}_{8} 30 \mathrm{p}$. Ioo ") et $\mathrm{B}_{1}$ (régime " $\mathrm{Tri} \mathrm{C}_{8} 25 \mathrm{p}$. Ioo ") par comparaison aux animaux homologues du lot $\mathrm{C}_{2}$ (régime "Glucides "). Par contre, elle a été réduite de $7 \mathrm{p}$. roo chez les animaux du lot $\mathrm{B}_{2}$ (régime " Tri $\mathrm{C}_{8} 25$ p.roo ")par comparaison à ceux du lot $\mathrm{C}_{3}$ (régime " Glucides ") et de $4 \mathrm{p}$. Ioo chez les animaux du lot $\mathrm{A}_{2}$ (régime " $\mathrm{Tri}_{8} 30 \mathrm{p}$. Ioo) par comparaison à ceux du lot $\mathrm{B}_{3}$ (régime "Tri $\mathrm{C}_{8} 25 \mathrm{p}$. IO0 ").

$\mathrm{L}$ 'acide caprylique induit, par ailleurs, une légère modification de la teneur des lipides corporels en acides gras saturés qu'elle accroît faiblement (tabl. 2) de I,9 à 2,7 points. Cet accroissement de la teneur des lipides corporels en acides gras saturés est entièrement imputable à l'accroissement de leur teneur en acides gras à chaîne moyenne (2,6 à 3 points) et en particulier en acide caprylique $(2,4$ à 3,5 points). Toutefois, com- 


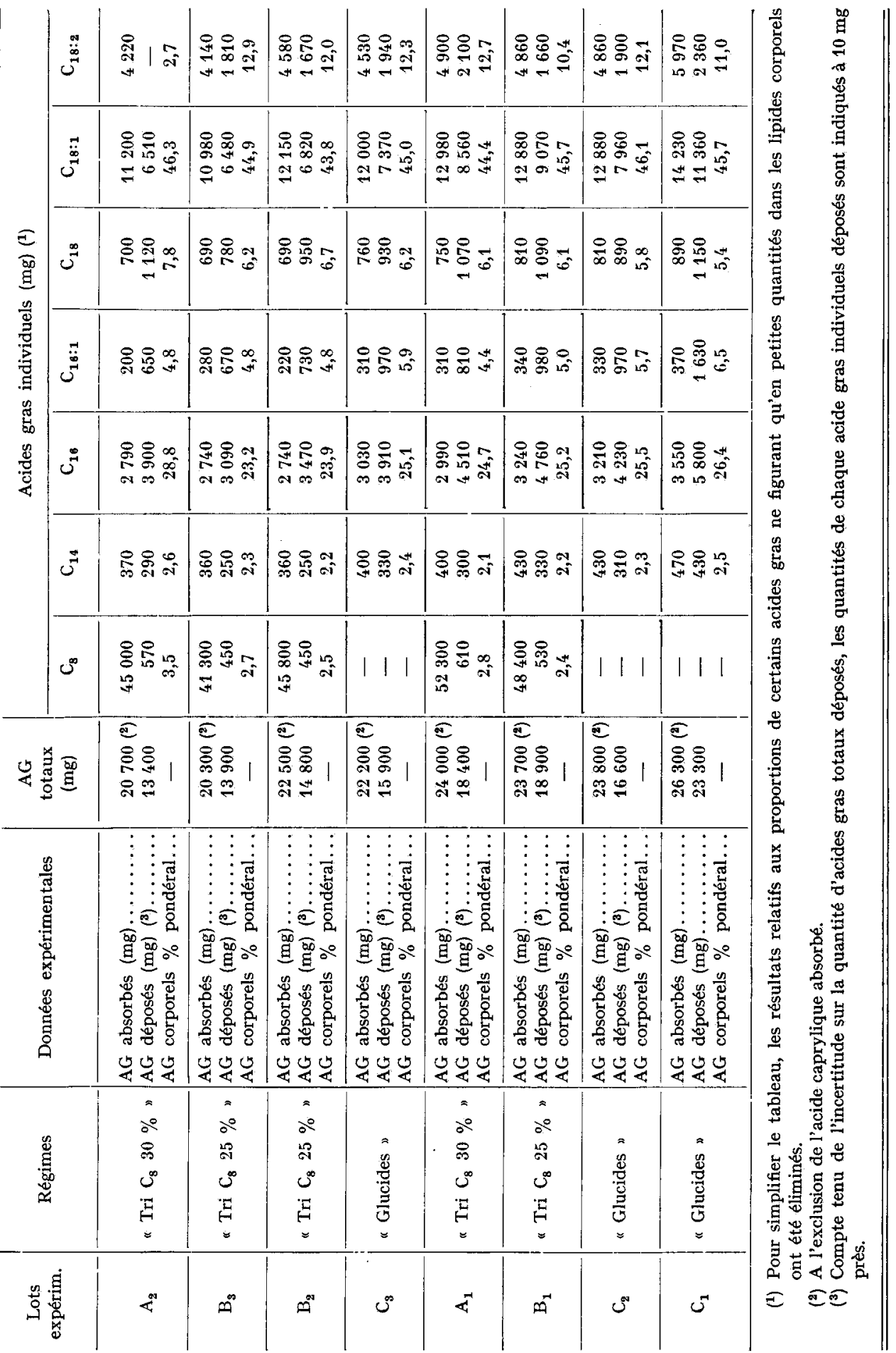


parativement aux résultats observés pour les animaux du lot $B_{3}$, la teneur des dépôts corporels en acides gras saturés est accrue significativement $(+8,3$ points) dans le cas des animaux du lot $\mathrm{A}_{2}$ ingérant le régime " $T \mathrm{ri} \mathrm{C}_{8} 30 \mathrm{p}$. I00", par accroissement de la teneur des dépôts en acides gras à chaîne moyenne ( $+I, I$ points) en acide palmitique $(+5,6$ points) et en acide stéarique ( $+\mathrm{I}, 6$ points), en compensation d'une diminution de la teneur des dépôts en acide linoléique (- ro,2 points).

A cette exception près, dans les conditions de l'expérience, 1'acide caprylique n'a apparemment aucune influence sur la teneur des dépôts en acides gras longs saturés. Cependant, si on rapporte la quantité de chaque acide gras déposée pendant la période expérimentale à la quantité absorbée dans le même temps, on peut mettre en évidence l'induction par l'acide caprylique d'un accroissement du dépôt d'acides palmitique et stéarique relativement au dépôt d'acide palmitoléique et oléique (tabl. 5). A cet égard, l'acide stéarique a un comportement particulier et le dépôt de cet acide est nettement accru dans tous les cas par l'acide caprylique. Pour l'ensemble des quatres acides l'influence de l'acide caprylique est particulièrement nette. Pour les lots $A_{2}$ et $B_{3}$, le rapport est en effet inchangé pour l'ensemble des deux acides gras insaturés $\left(C_{16: 1}\right.$ et $C_{18: 1}$ ) et accru de 30 points environ pour l'ensemble des deux acides gras saturés $\left(\mathrm{C}_{18}\right.$ et $\left.\mathrm{C}_{18}\right)$ lorsque la teneur de l'aliment en acide caprylique s'accroît de 5 points. Si l'on compare le lot témoin $C_{3}$ au lot expérimental $B_{2}$, l'influence de l'acide caprylique est moins nette : le rapport des quantités déposées aux quantités absorbées est réduit de 7 points environ pourr les insaturés et inchangés pour les saturés. Il en est de même si l'on compare le lot témoin $C_{2}$ respectivement aux lots expérimentaux $B_{1}$ et $A_{1}$, le rapport des quantités déposées aux quantités absorbées est accru respectivement de 8 et 4 points seulement pour les insaturés et de $I 7$ et de 22 points pour les saturés.

Au cours de la seconde expérience (tabl. 3) la quantité d'acides gras déposés pendant la période expérimentale est réduite de 16 p. Ioo par 1'introduction d'acide capry-

TABLEAU 3

Bilans d'énergie et d'acides gras observés au cours de l'expérience II

\begin{tabular}{l|c|c|c|c|c|c}
\hline Lots & Régimes & $\begin{array}{c}\text { EM ingérée } \\
\text { (kcal) }\end{array}$ & $\begin{array}{c}\text { E fixée } \\
\text { (kcal) }\end{array}$ & $\begin{array}{c}\text { E fixée } \\
\text { sous forme de } \\
\text { protéines (1) } \\
\text { (kcal) }\end{array}$ & $\begin{array}{c}\text { E fixée } \\
\text { sus forme de } \\
\text { lipides (1) } \\
\text { (kcal) }\end{array}$ & $\begin{array}{c}\text { E fixée } \\
\text { sous forme } \\
\text { d'AG (2) } \\
\text { (kcal) }\end{array}$ \\
\hline $\mathrm{D}$ & "Coprah Tri $\mathrm{C}_{8} 30 \% "$ & 1214 & 293 & 131 & 162 & 135 \\
\hline $\mathrm{E}$ & "Coprah 15\%" & 1217 & 285 & 135 & 151 & 121 \\
\hline $\mathrm{F}$ & "Tri $\mathrm{C}_{8} 15 \% "$ & 1200 & 283 & 140 & 143 & 121 \\
\hline $\mathrm{G}$ & "Glucides" & 1207 & 318 & 136 & 182 & 144 \\
\hline
\end{tabular}

(I) L'énergie fixée sous forme de protéines est obtenue par calcul à partir du bilan d'azote. Par différence avec le bilan énergétique on en déduit l'énergie fixée sous forme de lipides.

${ }^{2}{ }^{2}$ La quantité d'acides gras fixés a été déterminée par extraction et l'énergie correspondante calculée en adoptant une valeur de $9,5 \mathrm{kcal} / \mathrm{g}$ d'acide gras. 
EFFET MÉTABOLIQUE DE L'ACIDE CAPRYLIQUE
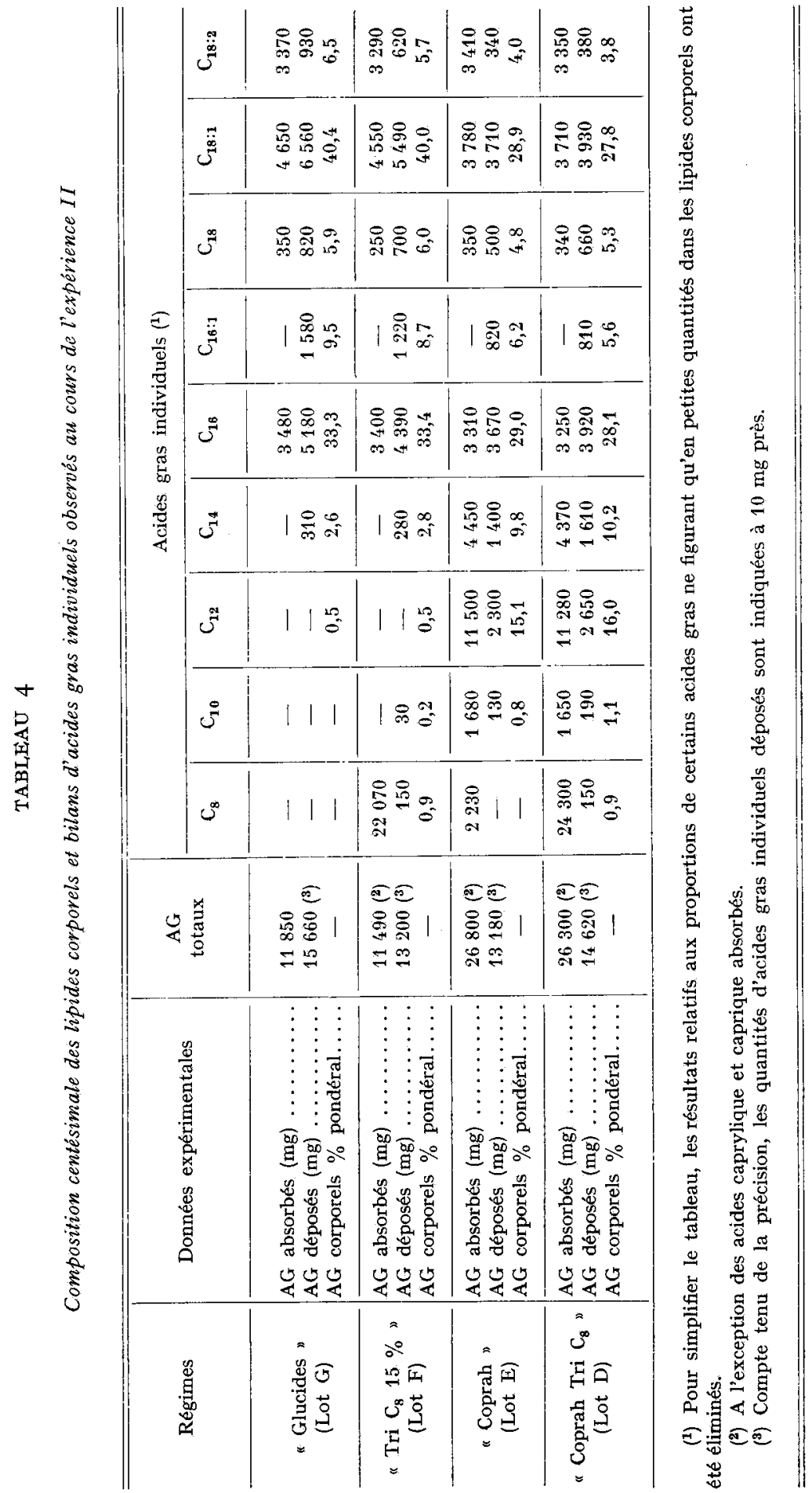


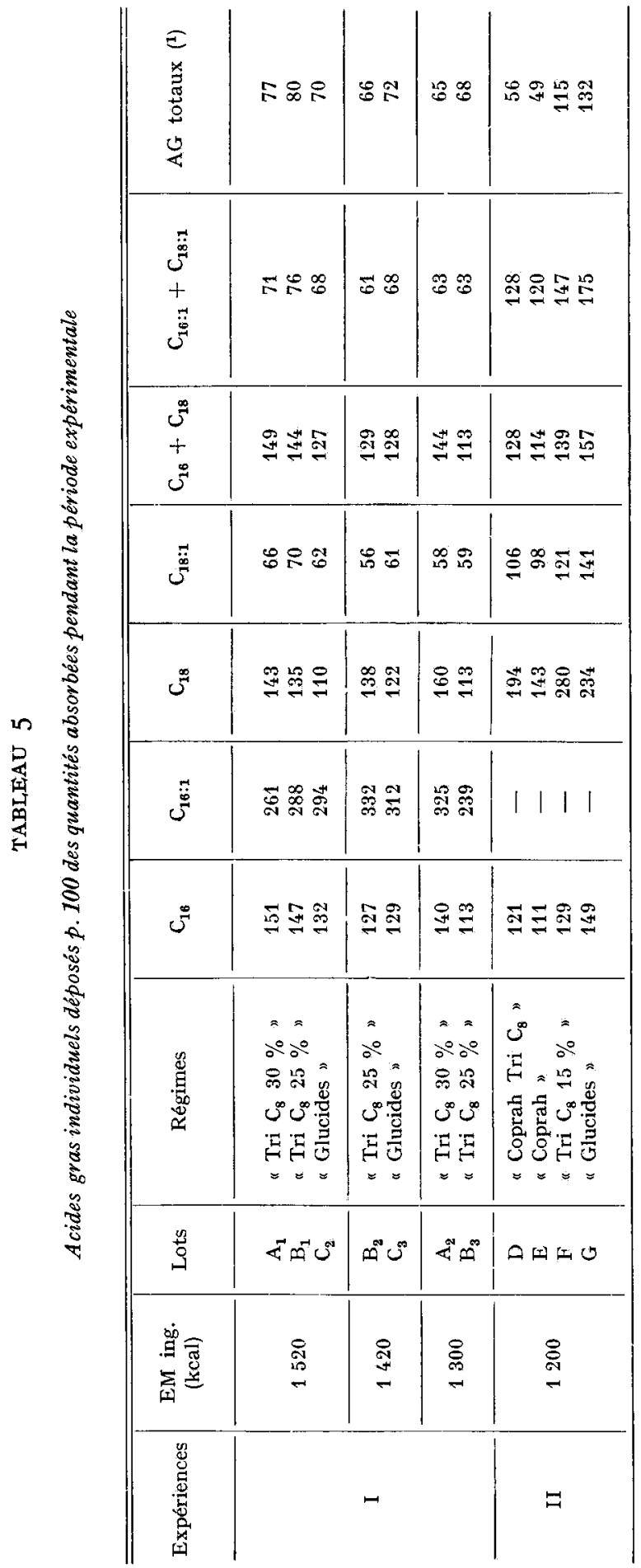


EFFET MÉTABOLIQUE DE L'ACIDE CAPRYLIQUE

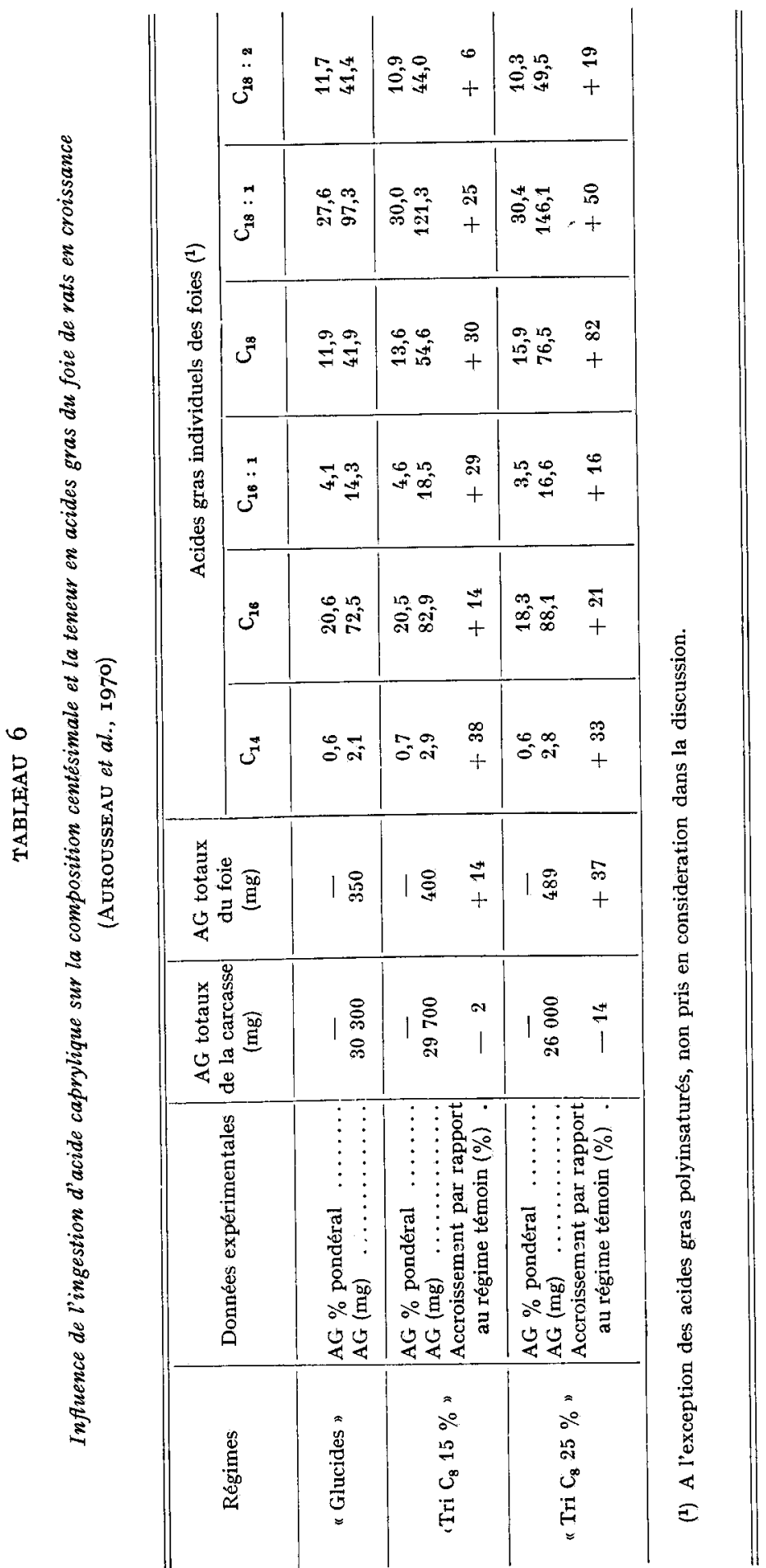

Annales de Biologie animale. - 1972. 
lique ou des acides gras à chaîne moyenne de 1'huile de coprah au niveau de 5 p. roo de 1'EM (lots $\mathrm{E}$ et $\mathrm{F}$ ) et de $7 \mathrm{p}$. Ioo environ par l'introduction du mélange de ces

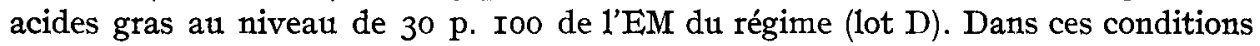
on peut remarquer (tabl. 4) que la teneur en acides gras saturés des lipides corporels passe de $42,3 \mathrm{p}$. roo chez les animaux recevant le régime témoin "glucides" (lot $\mathrm{G}$ ) à 43,8 p. Ioo chez les animaux recevant le régime "Tri $\mathrm{C}_{8} \mathrm{I}_{5} \mathrm{p}$. roo" (lot $\mathrm{F}$ ) à 59,5 p. roo chez les animaux recevant le régime "coprah I 5 p. Ioo " (lot E) et à $6 \mathrm{I}, 6$ p. I00 chez les animaux du lot $\mathrm{D}$. Pour ces deux derniers lots, l'accroissement de la teneur des lipides corporels en acides gras saturés est dû en majeure partie au dépôt d'acides laurique et myristique, ce qui confirme les résultats de LONGENECKER (I939) et de R. W. HARkins, et H. P. SARRETT (I968) : 24,9 p. Ioo chez les animaux du lot E et 26,2 p. Ioo chez les animaux du lot $D$.

Au cours de cette expérience, l'acide caprylique (lot F) n'exerce aucune influence sur la composition en acides gras des lipides corporels (tabl. 4 et 5). Les acides gras moyens de l'huile de coprah (lot $\mathrm{E}$ ), par contre, réduisent davantage les synthèses d'acides insaturés que celles d'acides saturés (tab1. 4 et 5) : le rapport des quantités déposées aux quantités absorbées est réduit de 55 points pour les premiers et de 33 points pour les seconds. L'adjonction d'acide caprylique aux acides gras moyens de l'huile de coprah (lot D) a pour effet d'accroître les synthèses apparentes d'acides gras (9 p. Ioo), la fixation d'acides gras saturés ( + r 3 p. roo) étant accrue davantage que la fixation d'acides gras insaturés ( 5 p. roo).

Dans les deux expériences, les régimes apportaient des quantités importantes d'acide linoléique ( $25 \mathrm{p}$. Ioo environ des acides gras longs) : on ne retrouve que de faibles quantités de cet acide dans les lipides corporels (Io à 35 p. Ioo des quantités ingérées).

Enfin, 1'acide caprylique accroît le poids du foie des animaux (+20 p. IOo) et leur contenu en acides gras de $+\mathrm{I} 4$ et $+37 \mathrm{p}$. roo respectivement pour les régimes "Tri $\mathrm{C}_{8} 15 \mathrm{p}$. Ioo " et " Tri $\mathrm{C}_{8} 25 \mathrm{p}$. Ioo ". La quantité totale d'acide palmitique contenue dans le foie des animaux est accrue, mais non sa teneur relative dans les lipides hépatiques.

\section{DISCUSSION}

Les résultats contradictoires concernant l'influence de l'acide caprylique sur la lipogenèse observés au cours de l'expérience I peuvent s'expliquer comme il est discuté dans la précédente communication (AURousseau, I972) par les différences de niveau de consommation (élevé, normal ou restreint). Dans ces conditions, en effet, le niveau de consommation modifie le rendement de l'utilisation de l'énergie des régimes pour l'ensemble des fonctions entretien et croissance ce qui se traduit par une modification des quantités d'énergie et de lipides fixés par les animaux expérimentaux.

L'infiuence favorable de l'acide caprylique sur le dépôt d'acides gras saturés relativement au dépôt d'acides gras insaturés peut être due en partie à l'accroissement des synthèses (KAUNITZ, Ig68 ; KRITCHEVSKY, I966-r967, BoL,IINGER, I965), 
mais semble principalement due à une action d'épargne exercée par l'acide caprylique sur les acides gras saturés à chaîne plus longue par diminution de leur catabolisme (BACH et al, I967), cette action d'épargne ne s'exerçant apparemment pas pour les acides gras insaturés (AUROusseAu et al., I970).

L'accroissement particulier des quantités d'acide stéarique retrouvées dans les lipides corporels consécutivement à l'ingestion de cet acide, montre que l'acide caprylique favorise les activités d'élongation relativement aux activités de synthèse, conformément aux observations de LEVEILLE et al., (I967) ou de DuCHEMIN et FAVARGER (I963).

Au cours de l'expérience I, l'effet de 1'acide caprylique est moins marqué pour les hauts niveaux d'ingestion : on peut rapprocher cette observation des travaux de GELLHORN et BENJAMIN (I965) qui montrent que la proportion d'acides insaturés formés croît avec l'intensité des synthèses d'acide gras.

L'absence à peu près totale d'influence de l'acide caprylique sur la composition en acides gras des lipides corporels, au cours de l'expérience II, peut s'expliquer par l'inhibition de la synthèse d'un acide gras par des apports exogènes de cet acide gras (BOLLINGER, I965 ; Bo'TTINO et REISER, I965 ; ZAKIM I965 ; BORTZ et LYNEN, I963).

L'accroissement du contenu en acide gras du foie de rats ayant ingéré des régimes comportant de l'acide caprylique, peut témoigner de synthèses lipidiques accrues et souligne le rôle important de cet organe dans le métabolisme des acides gras à chaîne moyenne. L'absence d'accroissement de la teneur en acide palmitique des lipides hépatiques relativement à leur teneur en acides insaturés entre en contradiction avec les résultats d'autres auteurs, mais peut s'expliquer par 1'accumulation dans le foie de l'acide oléique alimentaire (BOLIINGER, I965). Par ailleurs, le rapport $\epsilon$ tre les contenus des foies en acides palmitoléique et palmitique subit des variations faibles, mais tend à témoigner d'un accroissament des désaturations apparentes au niveau de cet organe avec le régime " $\operatorname{Tri}_{8} \mathrm{C}_{5} \mathrm{p}$. Ioo » et d'une réduction des désaturations apparentes avec le régime " $T \mathrm{Tri}_{8} 25 \mathrm{p}$. roo ". Les variations de la composition en acides gras des lipides corporels totaux indiquent une tendance inverse, les désaturations apparentes sont accrues du régime "Tri $\mathrm{C}_{8} \mathrm{I}_{5} \mathrm{p}$. Ioo " au régime "Trị $\mathrm{C}_{8} 25 \mathrm{p}$. Ioo" (AUROUSSEAU et al., 1970). Or les acides gras insaturés des tissus adipeux semblent avoir en grande partie une origine hépatique (CHRISTOphe et al., I969) : ces contradictions s'expliquent vraisemblablement par la succession de désaturations accrues et de remobilisation ou d'utilisation à des fins énergétiques d'intensité variable en fonction de la nature des acides gras (NAKAGAwA et Uchiyama, I968, I969, ; Dr Grorgio et al., I962; ELovson, I965). De plus, l'acide caprylique (observations non publiées) ou les acides gras à chaîne moyenne (AUROussEAU et al., 1972) exercent sur le métabolisme lipidique du Rat ou de 1'Agneau, des influences notablement différentes, vraisemblablement en raison de différences d'âge physiologique. Ces contradictions et ces différences soulignent les limites de la méthode de mesure de bilans d'acides gras chez le Rat et montrent que les " tendances " observées au cours de nos essais témoignent d'activités métaboliques dont l'importance peut varier selon l'espèce ou l'intensité de la croissance. 


\section{CONCLUSION}

La composition en acides gras des lipides corporels est la résultante de l'équilibre entre les apports exogènes d'acides gras et la succession de dégradations, de synthèses et interconversions intervenant dans divers organes au cours de la captation ou de la libération d'acides gras, de triglycérides ou de lipoprotéines. Fin conséquence, elle ne renseigne qu'imparfaitement sur le métabolisme lipidique des animaux.

Toutefois, la mesure du bilan d'acides gras au cours d'expériences mettant en jeu différents facteurs (âge des animaix, restrictions alimentaires, teneur des régimes en acides gras à chaîne moyenne, équilibre entre nutriments...) nous conduisent aux conclusions suivantes :

- Une part importante de 1'acide linoléique alimentaire est utilisée à des fins énergétiques.

- L'acide caprylique favorise le dépôt d'acide stéarique (élongation de l'acide palmitique).

- L'acide caprylique peut accroître l'importance des activités de synthèse relativement aux activités de désaturation, ou exercer une action d'épargne sur les acides laurique, myristique et palmitique. Ces effets peuvent être contrebalancés par de hauts niveaux d'alimentation ou par l'ingestion d'acides gras longs.

- Une part importante des acides caprique, laurique et myristique alimentaires est utilisée à des fins énergétiques. Toutefois ces acides se déposent dans les lipides corporels du rat en proportions plus importantes en présence d'acide caprylique. De plus ces acides favorisent l'accroissement de la quantité d'acide palmitique déposée dans les lipides corporels relativement à l'acide oléique.

Dans ces conditions, la présente étude du métabolisme lipidique de rats en croissance confirme l'intérêt que semblent présenter les acides gras à chaîne moyenne pour l'amélioration de la qualité des dépôts adipeux des jeunes animaux domestiques.

\section{Reçu pour publication en juin 1972.}

\section{SUMMARY}

\section{EFFECT OF CAPRYLIC ACID ON THE METABOLISM OF OTHER FATTY ACIDS, IN THE GROWING RAT}

\footnotetext{
A study of lipid metabolism as affected by caprylic acid has been done on male rats of the Vistar strain taken at $70 \mathrm{~g}$. body weight and previously employed to study energy utilization of this acid by the slaughter technique (AuRousseau, I972).

Experimental diets were built by substitution of caprylic acid for isocaloric quantities of the starch of a basal diet taken as a reference. Two levels of caprylic acid were tested in a first trial (25.8 and 28.2 p. roo of metabolizable energy of the diet) as well as three levels of intake ( " high", " normal ", or "restricted "). In a second trial, caprylic acid or lauric and myristic acid were introduced in the diet at the level of $15 \mathrm{p}$. Ioo of metabolisable energy and their interactions were tested by introducing each group of acids at this same level but together in the same diet.
} 
Caprylic acid intake, as already described, is followed by an increase of deposition in the carcass of medium-chain fatty acid, an increase of fatty acid synthesis and especially of fatty acid elongation relative to fatty acid desaturation. Moreover, caprylic acid favors the deposition of longer chain dietary fatty acids, apparently by protecting them from oxidative catabolism. In addition, caprylic acid effect may be over-shadowed by dietary long-chain fatty acids.

These findings are discussed in reference to intermediary metabolism and physiological age of experimental animals. Caprylic acid appears likely to favor the carcass quality of young growing domestic animals.

\section{RÉFÉRENCES BIBLIOGRAPHIQUES}

Aurousseau B., de Groot L., Bouvier J. C., Vermorel M., I97o, Utilisation métabolique des acides gras courts, moyens et longs par le rat en croissance. In "Energy metabolism of farm animals ". Proc. 5th Symp. held at Vitznau Steitzerland (ed. A. Schurch and C. Wenk), p. I8g.

Aurousseau B., I972. Utilisation énergétique et azotée de régimes comportant des acides caprylique, laurique et myristique par le rat en croissance. Influence du niveau d'alimentation. Ann. Biol. anim. Bioch. Biophys., $12(263-280)$.

Aurousseau B., Thériez M., Daniel Maryvonne, 1972. Influence de la nature des matières grasses incorporées dans l'aliment d'allaitement sur le métabolisme lipidique de l'agneau de boucherie. $A n n$. Biol. anim. Bioch. Biophys. (publication en cours).

Bach A., Metais P., Warter J. Comparaison par l'étude du ${ }^{14} \mathrm{CO}_{2}$ de l'air expiré, de l'utilisation de graines à acides gras longs, moyens et courts. Influence du support. C. R. Soc. Biol., 162, $247-25$ I.

Bezard J., Monneret-Boquillon M., ig66. Captation par le foie en perfusion des acides gras à . chaînes courtes et moyennes. Arch. sci. Physiol., 20, 359-379.

BOLLINGER J. N., I963. The metabolism of fatty acids derived from dietary triglycerides. The graduate school of the agricultural and mechanical college of Texas in partial fulfillment of the requirements for the degree of doctor of phylosophy (August r963).

Bollinger J. N., REISER R., 1965. The metabolic fate of fatty acids derived from dietary triglycerides. J.A. O. C. S., 42, II30-II33.

Bortz W. M., Lynenf, 1963. Elevation of long chain acyl CoA derivatives in livers of fasted rats. Biochem. Ztsch., 339, 77-82.

Bottino N. R., Anderson R. E., Reiser R., 1965. Dietary fatty acids : their metabolic fate and influence on fatty acid biosynthesis. J.A.O.C.S., 42, II24-II29.

Brady R. O., Gurin S., 1950. The biosynthesis of radioactive fatty acids ans cholesterol. J. Biol Chem., 186, 46I-469.

Clement J., Courel E., Klepping J., Briet S., 1964. Étude de la répartition chyloportale des acides gras à chaînes courtes et moyennes. J. Physiol., 18, 453-467.

Christophe J., Winand J., Furnelle J., I969. Distribution of newly synthesized fatty acids and metabolic heterogenety of NEFA and glycerides withem adipose tissue. Excepta Medica International Congress series n. 213. Proc. 8th intern. Congr. nutrition, Prague, Czechoslovakia.

De Groot L., Aurousseau B., Vermorel M., 1970. Influence de l'ingestion d'acide caprylique et d'acide oléique sur le métabolisme lipidique du rat en croissance. C. $R$. Acad. Sc., 270, 721-724.

Di Giorgio J., Bonanno R. A., Hegsted D. M., ig62. Effect of diet upon the in vitro metabolism of rat epididymal adipose tissue. $J$. Nutr., 78, 384-391.

Duchemin A., Favarger P., I963. Recherches sur la synthèse des acides gras. XI. Mode de synthèse de l'acide palmitique à partir de ses homologues inférieurs in vivo. Helv. Physiol. Acta, 21, $1-9$.

Elovson J., rg65. Conversion of palmitic acid ans stearic acid in the intact rat. Biochem. Biophys. Acta., 106, 201-203.

Gellhorn A., Benjamin N., 1965. Fatty acid biosynthesis and RNA function in fasting aging and diabetes. Adv. enz. Regul., 4, 19-26.

Harkins R. W., SARETT H. P., r968. Nutritional evaluation of medium chain triglycerides in the rat. J.A.O.C.S., 45, 23-26.

KaUnitz, Johnson $\quad$ R. E., 1968. Nutritional properties of MCT J. A. O.C. S., 45, 19-22.

KIRSChNer S. L., HARRis R. S., 1966. The influence of dietary fat on fatty acid biosynthesis in the rat. Biochem. Biophys. Acta., 116, I85-188.

KritcheVsky O., Shirley A., TePper, I967. The influence of MCT on cholesterol metabolism in rats. J. Nutr., 86, 67-72.

Leveille G. A., Pardini R. S., Tillotson J. A., I967. Influence of medium chain triglycerides on lipid metabolism in the chick. Lipids, 2, $46 \mathrm{I}-466$. 
Longenecker M; E., I939. Deposition and utilization of fatty acids of low molecular weight, and a fatty acid analysis of coconut oil. J. Biol. Chem., 130, 167-177.

Metais P., BACH A., I967. Vitesse d'oxydation relative des graines à acides gras à chaînes moyennes et courtes. Données préliminaires. Cahiers Nutr. Diététique, 2, 77-78.

Metais P., Bach A., Warter J., 1967. Comparaison par l'étude du ${ }^{14} \mathrm{CO}_{2}$ de l'air expiré de l'utilisation de graines à acides gras longs, moyens ou courts. $\quad C . R$. Soc., Biol., 161, I372.

Nagakawa M., Uchiyama M., I968. Comparison of desaturation of palmitic and stearic acids in rat liver preparation. J. Biochem., 68, 684-687.

Nakagawa M., Uchiyama M., I969. Esterification reaction affecting the pattern of mono-insaturated fatty acids in rat liver. J. Biochem., 6), 673-677.

Toulec R., Flanzy J., Rigaud J., ig68. Dosage des lipides des fèces. Ann. Biol. anim. Bioch. Biophys., 8, I8I-289.

ZAKIM D., I965. The effect of ethanol on hepatic fatty acid synthesis. Life Sci., 4, 209-2I4. 